\title{
Spectral domain optical coherence tomography
} as an effective screening test for hydroxychloroquine retinopathy (the "flying saucer" sign)

This article was published in the following Dove Press journal:

Clinical Ophthalmology

4 October 2010

Number of times this article has been viewed

\section{Eric Chen \\ David M Brown \\ Matthew S Benz \\ Richard H Fish \\ Tien P Wong \\ Rosa Y Kim \\ James C Major}

Retina Consultants of Houston, The Methodist Hospital, Houston, Texas, USA
Correspondence: Eric Chen

6560 Fannin Street, Suite 750

Houston, TX 77030, USA

Tel +l 7I3 5243434

Fax +I 7135243220

Email ecmd@houstonretina.com
Purpose: While the long-term incidence of hydroxychloroquine (HCQ) retinopathy is low, there remains no definitive clinical screening test to recognize HCQ toxicity before ophthalmoscopic fundus changes or visual symptoms. Patients receiving HCQ were evaluated with spectral domain optical coherence tomography (SD OCT) to assess the feasibility of identifying HCQ retinopathy at an early stage.

Methods: Twenty-five patients referred for the evaluation of hydroxychloroquine toxicity underwent a comprehensive ocular examination, Humphrey visual field (HVF) perimetry, time domain OCT, and SD OCT. Some patients with screening abnormalities also underwent further diagnostic testing at the discretion of the treating providers.

Results: Five patients were found to have SD OCT findings corresponding to HCQ toxicity and retinal damage as seen by clinical exam and/or HVF perimetry. Two patients with advanced toxicity were found to have significant outer retina disruption in the macula on SD OCT. Three patients with early HCQ toxicity and HVF 10-2 perifoveal defects were found to have loss of the perifoveal photoreceptor inner segment/outer segment (IS/OS) junction with intact outer retina directly under the fovea, creating the "flying saucer" sign. While two of these three patients had early ophthalmoscopic fundus changes, one had none.

Conclusion: Outer retinal abnormalities including perifoveal photoreceptor IS/OS junction disruption can be identified by SD OCT in early HCQ toxicity, sometimes even before ophthalmoscopic fundus changes are apparent. SD OCT may have a potential complementary role in screening for HCQ retinopathy due to its quick acquisition and because it is more objective than automated perimetry.

Keywords: drug toxicity, hydroxychloroquine, photoreceptors, screening test, spectral domain optical coherence tomography

\section{Introduction}

The use of hydroxychloroquine (HCQ), an antimalarial drug utilized for a range of rheumatologic and dermatologic diseases, is associated with a low incidence of retinopathy $(0.5 \%)$ when used at recommended doses $(<6.5 \mathrm{mg} / \mathrm{kg}$ per day $) .{ }^{1}$ It has largely replaced chloroquine because of decreased ocular toxicity at high dosages, ${ }^{2}$ but can still lead to potentially irreversible visual loss. Although multiple modalities have been proposed for the screening, there remains no "gold standard" for the identification of HCQ toxicity before the onset of decreased vision or ophthalmoscopic fundus changes. ${ }^{3}$ In 2002 , the American Academy of Ophthalmology published preferred practice patterns for HCQ retinopathy screening. Recommendations included a comprehensive eye exam and Humphrey visual field (HVF) (Humphrey Instruments Inc., San Leandro, CA, USA) 
10-2 perimetry or Amsler grid testing, with other testing left to the discretion of the provider. Follow-up was based on risk factors including dosage, duration of use, body habitus, liver or renal function, concomitant retinal disease, and age. However, it was acknowledged that even with screening, severe vision loss is still possible. ${ }^{4}$ At the present time, it is still not known whether the crucial factor in developing toxicity is daily dose, duration of usage, or cumulative dose. ${ }^{5}$

Patients may present with a range of complaints; while some are asymptomatic, others will have problems with central vision or field defects, glare, night blindness, or deficiencies in color vision. ${ }^{6,7}$ Clinical changes tend to be a late finding in HCQ retinopathy and are characterized by bilateral pigmentary macular changes, often sparing the foveal center and leading to a characteristic "bull's eye" maculopathy. Paracentral scotomas on HVF 10-2 perimetry correlate well with retinal damage and may appear before other clinical findings are apparent. ${ }^{8}$ Color vision testing defects and abnormalities in fluorescein angiography (FA) and electroretinography (ERG) can also be seen. ${ }^{6,9,10}$ The exact mechanism of retinal toxicity is unknown; previous studies have implicated damage to outer retinal structures ${ }^{11,12}$ such as photoreceptors and retinal pigment epithelium cells, while others have suspected damage to retinal ganglion cells, the inner plexiform layer, or the retinal nerve fiber layer. ${ }^{13-15}$

Recently, a research prototype of an ultra-high resolution optical coherence tomography (OCT) system identified distinctive abnormalities in the perifoveal photoreceptor inner segment/outer segment (IS/OS) junction in symptomatic patients with HCQ toxicity and findings on visual fields and multifocal ERG. ${ }^{16}$ Patients with more advanced retinopathy were also found to have thinning of the outer nuclear layer. Our study was designed to evaluate the utility of commercially available spectral domain OCT (SD OCT) systems for their ability to detect early retinal changes due to HCQ toxicity.

\section{Methods}

Charts from consecutive patients referred for the evaluation of HCQ retinopathy were reviewed retrospectively. All patients underwent a comprehensive eye examination, HVF 10-2 perimetry, time domain OCT imaging (Stratus; Carl Zeiss Meditec, Inc., Dublin, CA, USA), and SD OCT imaging with both the Heidelberg Spectralis HRA + OCT (Spectralis; Heidelberg Engineering, Heidelberg, Germany) and Cirrus HD-OCT (Cirrus; Carl Zeiss Meditec, Inc., Dublin, CA, USA). Based on the results of the above screening examinations and per the discretion of the treating physician, some patients also had fundus autofluorescence imaging (FAF), color fundus photography, FA, and multifocal electroretinogram testing. Charts were reviewed for HCQ dose and duration, indication for HCQ use, clinical exam findings, and imaging results. The Institutional Review Board of The Methodist Hospital in Houston, TX, approved the study protocol.

\section{Results}

Twenty-five patients (one male, 24 female, mean age of 55 with range of 28-78) were referred for the evaluation of HCQ retinopathy (Table 1). Indications for HCQ usage included: 12 for systemic lupus erythematosus, six for rheumatoid arthritis, three for lichen planopilaris, two for Sjogren's syndrome, and one each for scleroderma, dermatomyositis, and unspecified antinuclear antibody positive disease. For this study we included all patients referred to our practice for evaluation of HCQ retinopathy; several patients were referred for "baseline" examinations, and the longest duration of treatment was 22 years. Most patients were on standard dosages of 200 or $400 \mathrm{mg}$ daily, but when bodyweight was taken into consideration, seven of the 25 were on dosages higher than the recommended $6.5 \mathrm{mg} / \mathrm{kg} /$ day. Cumulative dosages of HCQ excluding those patients seen for baseline evaluations ranged from 146 to $2920 \mathrm{~g}$.

Nine of the 25 patients were found to have funduscopic changes suspicious for HCQ toxicity, ranging anywhere from mild RPE changes to advanced bull's eye maculopathy, while 16 patients presented without any clinical signs of HCQ toxicity. Two patients who presented with macular pucker and one who presented with drusen alone were not considered to have retinal changes consistent with possible HCQ retinopathy. Out of the nine patients with funduscopic abnormalities, four had no defects on either HVF 10-2 perimetry or SD OCT testing. The remaining five patients all had visual field defects on HVF 10-2 perimetry, and four of these had abnormalities on SD OCT. Of the 16 patients with normal funduscopic exams, eight patients had HVF 10-2 abnormalities while only one had changes on SD OCT.

In patients with early HCQ retinopathy, SD OCT imaging showed loss of the perifoveal photoreceptor IS/OS junction, perifoveal thinning of the outer nuclear layer, and an apparent posterior displacement of the inner retinal structures toward the retinal pigment epithelium. This displacement led to variable loss of the normal foveal depression, and when combined with preservation of an intact photoreceptor IS/OS junction within the foveal region, created a "flying saucer" sign (Figure 1). We have chosen the term "flying saucer", a well recognized shape in the popular media, to describe the apparent ovoid appearance of the central fovea created by 


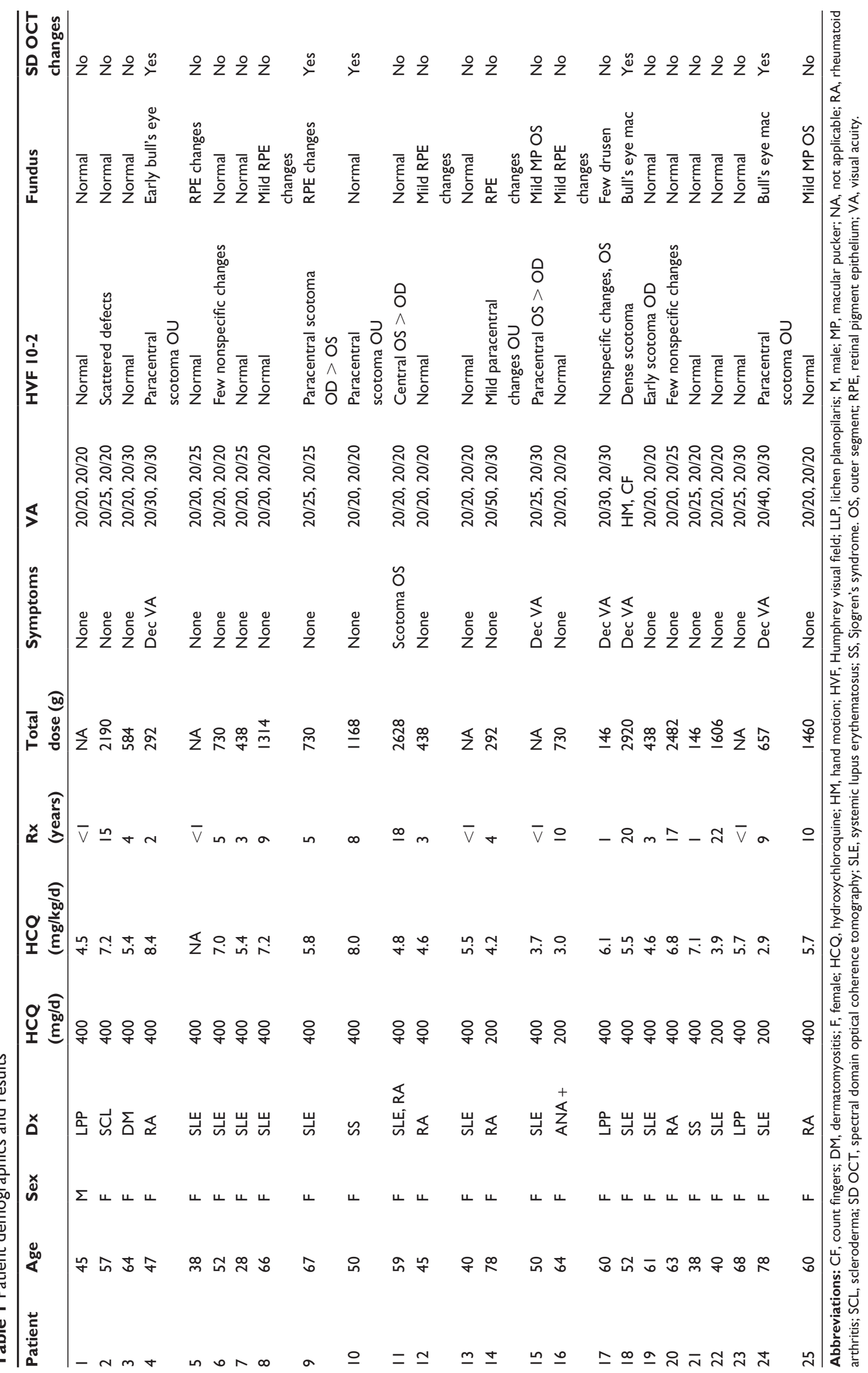




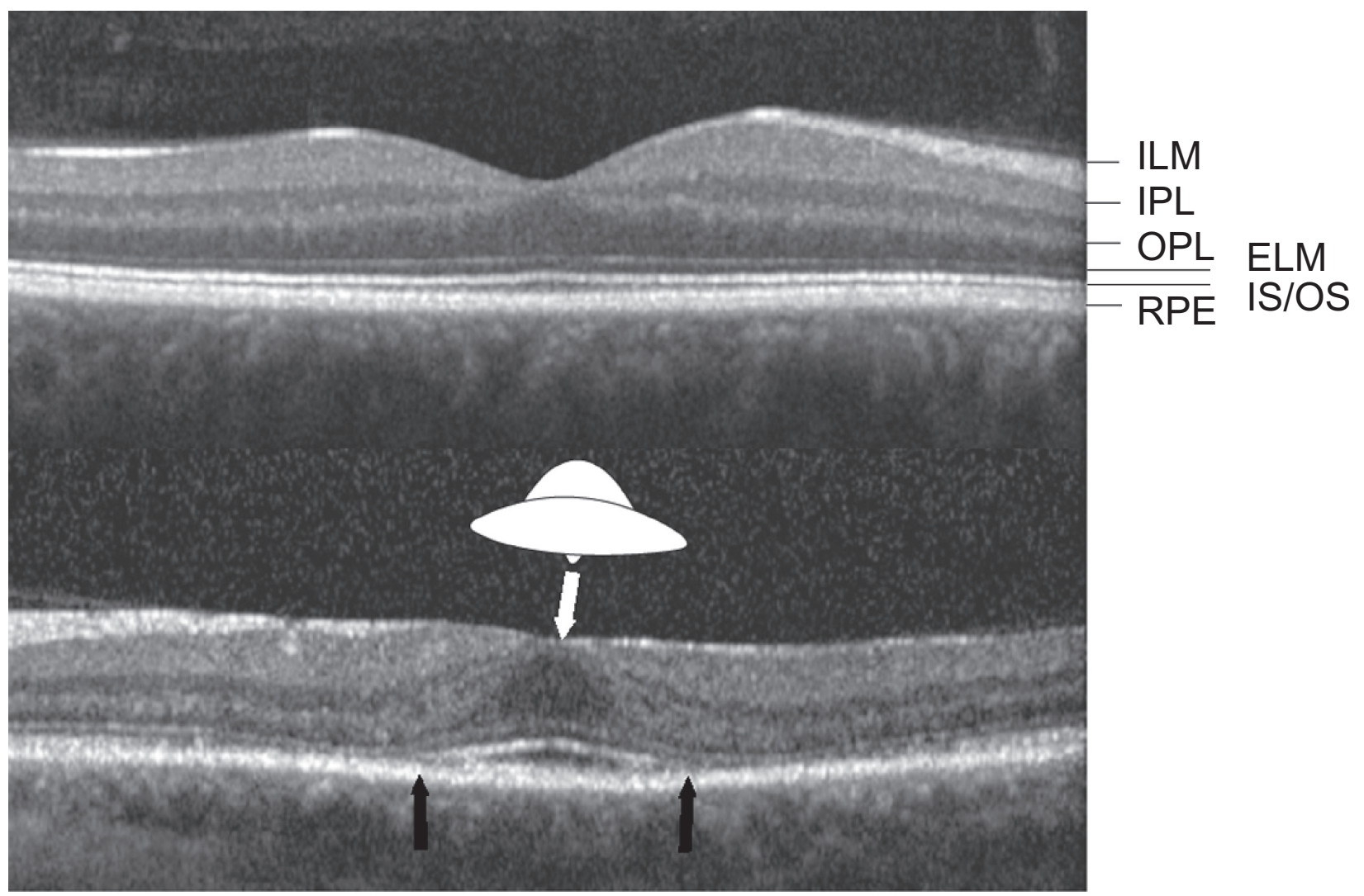

Figure I Top: Normal Spectralis spectral domain optical coherence tomography (SD OCT) image with intact photoreceptor inner segment/outer segment junction (IS/ OS). Bottom: Spectralis SD OCT from the left eye of patient 10 showing the "flying saucer" sign of hydroxychloroquine retinopathy, an ovoid appearance of the central fovea created by preservation of central foveal outer retinal structures (seen between the black arrows) surrounded by perifoveal loss of the photoreceptor IS/OS junction, and perifoveal outer retinal thinning.

Abbreviations: ILM, internal limiting membrane; IPL, inner plexiform layer; OPL, outer plexiform layer; ELM, external limiting membrane; RPE, retinal pigment epithelium.

preservation of the outer retinal structures in the central fovea surrounded by perifoveal loss of the photoreceptor IS/OS junction and perifoveal outer retinal thinning.

Five of the 25 patients had abnormal SD OCT findings that correlated with exam and/or HVF perimetry findings suspicious for HCQ retinopathy. Duration of treatment with HCQ in these patients ranged from 2 to 20 years, HCQ daily dose adjusted for bodyweight ranged from 2.9 to $8.4 \mathrm{mg} / \mathrm{kg}$, and cumulative dose ranged from 292 to $2920 \mathrm{~g}$. Three of the five patients were aware of vision changes, and while four had abnormalities on funduscopic exam, one of the patients had a normal ophthalmologic fundus exam. All five patients did have abnormalities on HVF 10-2 perimetry, ranging from paracentral to dense scotomas. In the five patients with SD OCT abnormalities, 3 had signs of early HCQ retinopathy while 2 had advanced changes. The "flying saucer" sign was present in all three of the five patients who presented with early HCQ retinopathy.

Two patients presented with advanced HCQ retinopathy and moderate to severe vision loss. Funduscopic findings ranged from pronounced bull's eye maculopathy to severe macular atrophy. SD OCT findings ranged from disruption of outer retinal integrity with preservation of foveal IS/OS photoreceptors to complete structural disorganization of the outer retina.

Normal SD OCT images with preservation of perifoveal outer retinal structures were seen in 20 of the 25 patients. Duration of treatment with HCQ in these patients ranged from less than one year to 22 years, daily doses from 3.0 to $7.2 \mathrm{mg} / \mathrm{kg}$, and cumulative doses from 146 to $2628 \mathrm{~g}$. Of these patients with no SD OCT abnormalities, three complained of vision changes and eight had abnormalities on HVF 10-2 perimetry.

\section{Discussion}

Although it is better tolerated at high dosages than chloroquine and infrequently causes retinopathy, HCQ can still cause severe irreversible vision loss. HCQ screening for identification of early toxicity is critical to allow for cessation of the drug and potential prevention of vision loss. ${ }^{17}$ The American Academy of Ophthalmology preferred practice 
patterns recommend screening initially for HCQ toxicity with a complete ophthalmic exam and either HVF 10-2 perimetry or Amsler grid testing, with other testing optional and at the discretion of the provider., ${ }^{4,18}$ While HVF perimetry and Amsler grid testing may show abnormalities even before the onset of funduscopic changes, ${ }^{8}$ they are still limited in that they only detect toxicity after significant retinopathy has occurred. Furthermore, Amsler grid testing is very subjective, and automated visual field perimetry has a steep learning curve and can be inconclusive. Clinical exam findings can be subtle and tend to be a late finding.

Multiple other modalities have been suggested for HCQ toxicity screening. Color testing may help detect early maculopathy, ${ }^{4}$ and FA may help in patients who have difficulty with visual field testing. ${ }^{17}$ Fundus autofluorescence may detect early retinal pigment epithelium alterations, ${ }^{19}$ and preferential hyperacuity perimetry testing may pick up defects in early hydroxychloroquine toxicity. ${ }^{20}$ Several other reports suggest that serial multifocal ERGs may detect decreased retinal function even earlier than other modalities in patients with otherwise normal clinical examinations. ${ }^{21-24}$ To date, however, many of these tests are not widely and readily available, and there still remains a lack of consensus on which diagnostic test is the most useful.

Rodriguez-Padilla et al $^{16}$ first described some of the findings in HCQ toxicity on a research prototype ultra-high resolution OCT machine, and other recent case reports have reported HCQ retinopathy findings on SD OCT machines. ${ }^{25,26}$ In our study, SD OCT abnormalities due to HCQ toxicity were identified in five patients when using both SD OCT systems. As expected, two patients with advanced HCQ retinopathy had SD OCT findings that demonstrated loss of outer retinal integrity and structural disorganization that correlated with advanced bull's eye maculopathy and/or macular atrophy seen on clinical, angiographic, and with visual field testing. Three other patients with earlier stages of HCQ toxicity identified by SD OCT all presented with paracentral scotomas seen on HVF 10-2 testing usually associated with HCQ retinopathy. All three demonstrated characteristic perifoveal outer retinal abnormalities seen on SD OCT that we have termed the "flying saucer" sign; these findings include preservation of the outer retinal structures in the central fovea, perifoveal loss of the photoreceptor IS/OS junction and outer retinal thinning, posterior or "sinkhole" displacement of the inner retinal structures toward the retinal pigment epithelium, ${ }^{25}$ and variable loss of the normal foveal depression.

While the "flying saucer" sign we have described is not pathognomonic nor even necessary for the diagnosis of HCQ retinopathy, it may be helpful in questionable or inconclusive cases when reviewed in the context of clinical findings as well as other diagnostic testing. This "flying saucer" may not even represent the earliest stage of HCQ toxicity visible on SD OCT, as Stepien et al described a "preclinical" stage of HCQ toxicity where the photoreceptor IS/OS junction appears "moth-eaten" due to preferential loss of cone photoreceptors. ${ }^{25}$ Even though the "flying saucer" sign was poorly visualized using time domain OCT that was also performed in all of the patients, this perifoveal loss of the photoreceptor IS/OS junction and
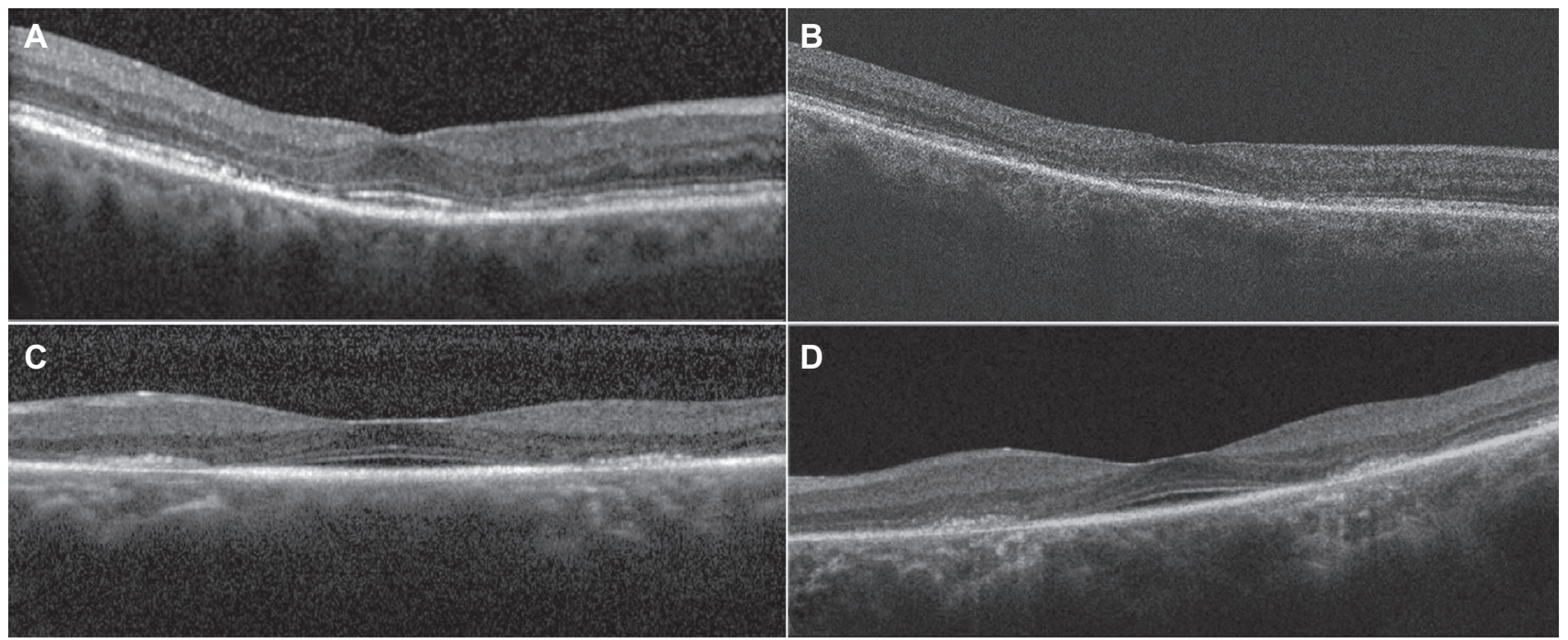

Figure 2 Spectral domain optical coherence tomography (SD OCT) images taken with different commercially available SD OCT machines demonstrate the "flying saucer" sign is consistent in different individuals with hydroxychloroquine retinopathy. A) Heidelberg Spectralis SD OCT in patient 9, OD. B) Zeiss Cirrus SD OCT in patient 9, OD. C) Heidelberg Spectralis SD OCT in patient 4, OS. D) Zeiss Cirrus SD OCT in patient 4, OS. 
outer retinal structures was easily visualized and appeared consistently in scans across both the Heidelberg Spectralis SD-OCT and the Zeiss Cirrus SD-OCT, both commercially available OCTs found in many retinal offices (Figure 2).

Patient 4 presented with subtle bull's eye retinopathy seen on both clinical fundus exam and angiographic testing and HVF with paracentral scotomas OU; although she had a prior history of intermittent HCQ use for a total duration of only 2 years over a period of 4 years, she had an adjusted HCQ daily dose of $8.4 \mathrm{mg} / \mathrm{kg}$ and a "flying saucer" on SD OCT helped confirm HCQ retinopathy (Figure 2B). A national database of over 4000 HCQ patients has found only a few per 1000 with probable toxicity in the first 5-7 years of usage (personal communication, Marmor), although another review has noted HCQ retinopathy in a patient treated for as little as 1.9 months. ${ }^{6}$ Her symptoms appeared to progress even after the cessation of HCQ in 2007, which has been previously reported in chloroquine toxicity, ${ }^{27}$ although her clinical and SD OCT findings have been stable since we first saw her over one year ago in 2009.

In one of the patients with early HCQ retinopathy, the SD OCT imaging was particularly helpful in making a timely diagnosis of toxicity. Patient 10 (Figure 3) was found to have a completely normal fundus exam, autofluorescence, and fluorescein angiography. Her time domain OCT was unremarkable, but SD OCT demonstrated the "flying saucer" sign and thinning of the outer nuclear layer in both eyes. HVF 10-2 showed classic paracentral scotomas that had progressed significantly from the nonspecific changes that were seen in the previous year's exam; no SD OCT had been performed at that time. Although no other clinical or ancillary testing was able to detect toxicity in this patient, SD OCT findings helped confirm HVF findings. These SD OCT findings were maintained 12 months after cessation of HCQ with persistence of the "flying saucer".

A careful review of the abnormal SD OCTs in this patient and others in the study also demonstrated that SD OCT testing could show a spectrum of findings that correspond to progression between different stages of HCQ toxicity. Figure 4A shows patient 24, who presented with moderate vision loss and clinically significant bull's eye maculopathy that was even more apparent on FAF; a small layer of intact foveal IS/OS photoreceptors allowed her to maintain 20/40 visual acuity. Figure 4B shows patient 18, who had advanced foveal atrophy, dense paracentral scotomas, and complete outer retinal disruption on SD OCT; her vision was hand motions. These progressive SD OCTs also reveal an increasing visualization of the choriocapillaris due to retinal atrophy with progressively increasing levels of toxicity.
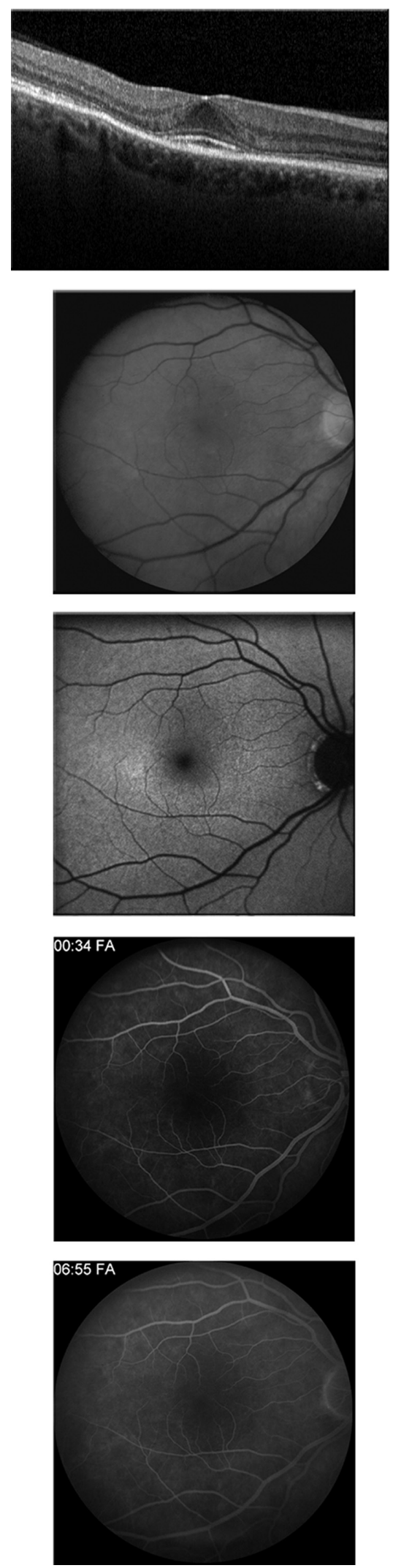

Figure 3 Spectral domain optical coherence tomography (SD OCT), red-free photo, fundus autofluorescence, and early and late fluorescein angiogram in patient 10, who had no symptoms, normal clinical exam and fundus autofluorescence imaging, Humphrey visual field 10-2 with paracentral scotomas, and "flying saucer" sign seen on SD OCT. 

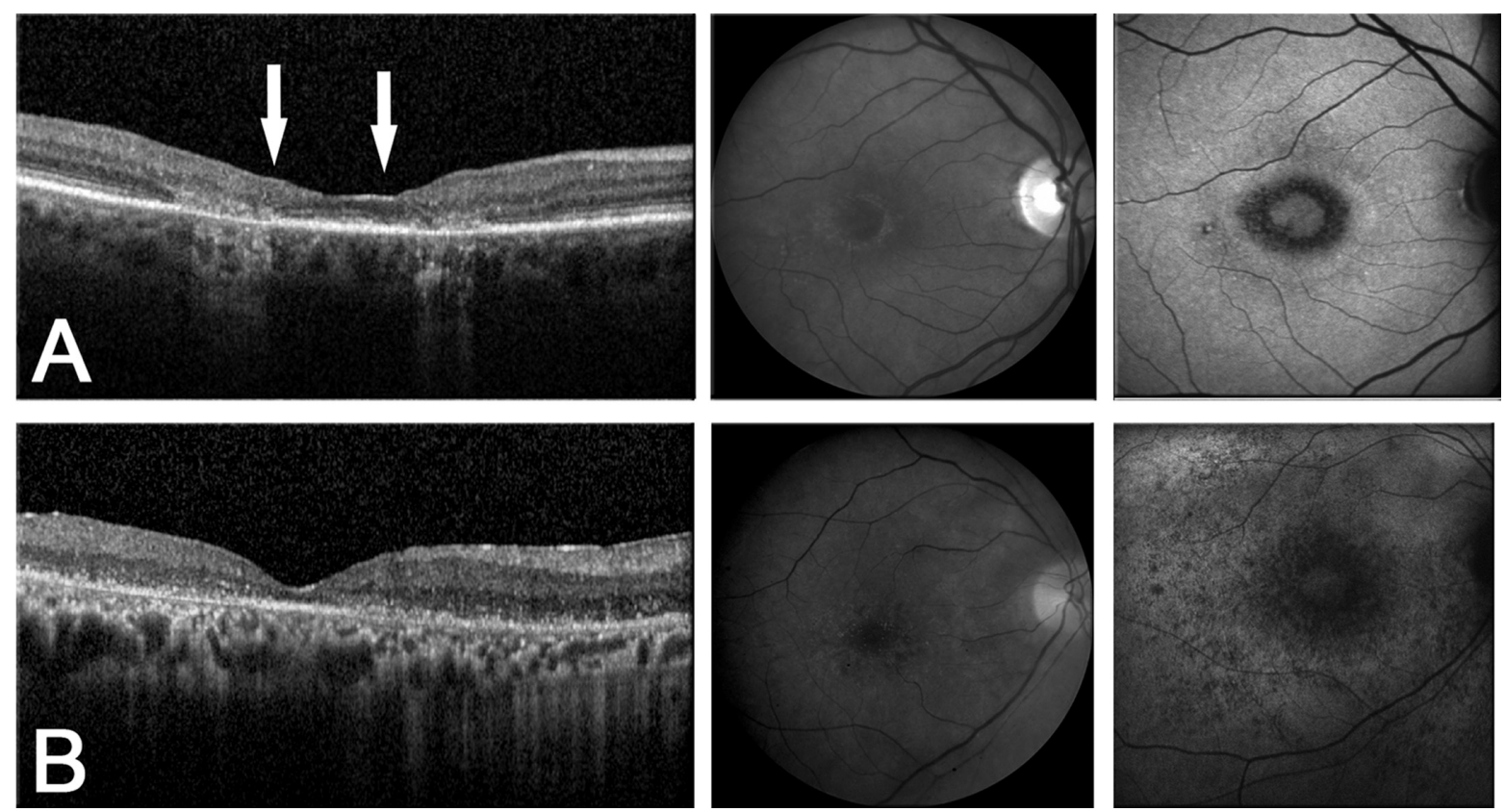

Figure 4 Spectral domain optical coherence tomography (SD OCT) images, red-free photos, and fundus autofluorescence (FAF) demonstrate a spectrum of findings seen with different stages of hydroxychloroquine retinopathy. Note the increased signal of choroidal vessels on SD OCT as the retina becomes increasingly atrophic with progressive toxicity starting from early toxicity in patient 10 seen in Figure 3. A) Moderate toxicity. Patient 24: vision loss, clinical bull's eye maculopathy even more apparent on FAF, Humphrey visual field (HVF) 10-2 paracentral scotomas, and significant perifoveal outer retinal dropout but preservation of central photoreceptor inner segment/ outer segment junction (between the arrows) seen on SD OCT allowing 20/40 vision. B) Late toxicity. Patient I8: severe vision loss to hand motion, pronounced clinical and FAF bull's eye maculopathy, dense HVF 10-2 paracentral scotomas, and complete disruption of outer retinal structures on SD OCT.

In the patient with the normal funduscopic exam, changes on SD OCT imaging allowed us to detect HCQ retinopathy that was suggested by HVF but could have otherwise gone undetected on clinical examination alone. A lack of SD OCT findings was also helpful in ruling out true HCQ toxicity when ophthalmoscopic fundus exam or other imaging modalities including HVF were inconclusive. As toxicity can present as a spectrum of disease, any macular changes or abnormalities seen on HVF in a patient on HCQ therapy needs to be further evaluated for possible retinopathy. Upon further review, of the nine patients who had abnormal funduscopic exams even potentially attributable to HCQ retinopathy, only the four with both SD OCT and HVF changes were deemed to have true HCQ retinopathy. Of the 15 other individuals with normal funduscopic findings, eight had abnormalities on HVF perimetry; aside from patient 10 discussed previously, when these "abnormal" HVF cases where analyzed together with their accompanying normal SD OCT findings, none of the patients were judged clinically to have signs of HCQ toxicity.

For patients being treated with HCQ, SD OCT may play an important potential role in screening, as there remains a lack of a true "gold" standard in detecting HCQ retinopathy before vision loss has occurred. Clinical funduscopic findings can be very subtle and tend to be a late finding, and
Amsler grid findings can be very subjective. HVF perimetry has a steep learning curve and can be very difficult for some patients, leading to nonspecific and inconclusive results. In addition, most retina specialists do not have HVF in their offices and HVF may require separate scheduling to allow for an undilated test. Other modalities such as multifocal ERGs ( $m E R G$ ) have been shown to potentially identify HCQ toxicity changes at an earlier stage; however, mERGs are expensive, require special training for administration and interpretation, and are not readily available.

This study demonstrates that unique SD OCT findings in the retina can identify early HCQ retinopathy using commercially available SD OCT systems and may play a complementary role in screening patients in addition to a comprehensive eye exam and HVF testing. SD OCT imaging allows for anatomical changes to be identified, eliminating potential errors from poor patient participation and diminishing the need for further testing when nonspecific changes are identified either on clinical exam or HVF testing. Characteristic perifoveal photoreceptor IS/OS junction loss and thinning of outer retinal structures in areas of early HCQ toxicity can be identified on SD OCT, leading to the "flying saucer" sign in the central fovea. A lack of SD OCT changes can also be useful in further evaluating cases of questionable fundus or visual field changes. Given the increasing availability of SD 
OCT in many retina practices, the ease of image acquisition, the lack of subjective findings, and the ability to register and compare images from visit to visit, SD OCT may prove to be an effective, reliable, and efficient tool to add to the protocol for HCQ toxicity screening.

\section{Summary statement}

Spectral domain optical coherence tomography may have a role in the detection of early hydroxychloroquine retinopathy because of its short acquisition time and greater objectivity relative to automated visual field testing. Perifoveal photoreceptor inner segment/outer segment junction disruption (the "flying saucer" sign) can be used to detect early toxicity, sometimes even before the appearance of clinical fundus changes.

\section{Disclosure and acknowledgment}

None of the authors have any proprietary interest in any of the data. Preliminary data from this study was presented at Retina Congress 2009.

The authors would like to acknowledge Kim E Stepien, $\mathrm{MD}$, for her contributions in preparing and editing this manuscript.

\section{References}

1. Mavrikakis I, Sfikakis PP, Mavrikakis E, et al. The incidence of irreversible retinal toxicity in patients treated with hydroxychloroquine: a reappraisal. Ophthalmology. 2003;110(7):1321-1326.

2. Levy GD, Munz SJ, Paschal J, Cohen HB, Pince KJ, Peterson T. Incidence of hydroxychloroquine retinopathy in 1,207 patients in a large multicenter outpatient practice. Arthritis Rheum. 1997;40(8):1482-1486.

3. Lee AG. Hydroxychloroquine screening. Br J Ophthalmol. 2005;89(5): 521-522.

4. Marmor MF, Carr RE, Easterbrook M, Farjo AA, Mieler WF. Recommendations on screening for chloroquine and hydroxychloroquine retinopathy: a report by the American Academy of Ophthalmology. Ophthalmology. 2002;109(7):1377-1382.

5. Marmor MF. The dilemma of hydroxychloroquine screening: new information from the multifocal ERG. Am J Ophthalmol. 2005;140(5): 894-895.

6. Yam JC, Kwok AK. Ocular toxicity of hydroxychloroquine. Hong Kong Med J. 2006;12(4):294-304.

7. Tehrani R, Ostrowski RA, Hariman R, Jay WM. Ocular toxicity of hydroxychloroquine. Semin Ophthalmol. 2008;23(3):201-209.

8. Elder M, Rahman AM, McLay J. Early paracentral visual field loss in patients taking hydroxychloroquine. Arch Ophthalmol. 2006;124(12): 1729-1733.

Clinical Ophthalmology

\section{Publish your work in this journal}

Clinical Ophthalmology is an international, peer-reviewed journal covering all subspecialties within ophthalmology. Key topics include: Optometry; Visual science; Pharmacology and drug therapy in eye diseases; Basic Sciences; Primary and Secondary eye care; Patient Safety and Quality of Care Improvements. This journal is indexed on Submit your manuscript here: http://www.dovepress.com/clinical-ophthalmology-journal
9. Klinger G, Morad Y, Westall CA, et al. Ocular toxicity and antenatal exposure to chloroquine or hydroxychloroquine for rheumatic diseases. Lancet. 2001;358(9284):813-814.

10. Jones SK. Ocular toxicity and hydroxychloroquine: guidelines for screening. Br J Dermatol. 1999;140(1):3-7.

11. Duncker G, Schmiederer M, Bredehorn T. Chloroquine-induced lipidosis in the rat retina: a functional and morphological study. Ophthalmologica. 1995;209(2):79-83.

12. Duncker G, Bredehorn T. Chloroquine-induced lipidosis in the rat retina: functional and morphological changes after withdrawal of the drug. Graefes Arch Clin Exp Ophthalmol. 1996;234(6):378-381.

13. Hallberg A, Naeser P, Andersson A. Effects of long-term chloroquine exposure on the phospholipid metabolism in retina and pigment epithelium of the mouse. Acta Ophthalmol (Copenh). 1990;68(2):125-130.

14. Pasadhika S, Fishman GA. Effects of chronic exposure to hydroxychloroquine or chloroquine on inner retinal structures. Eye (Lond). 2010; 24(2):340-346.

15. Pasadhika S, Fishman GA, Choi D, Shahidi M. Selective thinning of the perifoveal inner retina as an early sign of hydroxychloroquine retinal toxicity. Eye (Lond). 2010;24(5):756-762;quiz 763.

16. Rodriguez-Padilla JA, Hedges TR 3rd, Monson B, et al. High-speed ultra-high-resolution optical coherence tomography findings in hydroxychloroquine retinopathy. Arch Ophthalmol. 2007;125(6):775-780.

17. Easterbrook M. Detection and prevention of maculopathy associated with antimalarial agents. Int Ophthalmol Clin. 1999;39(2):49-57.

18. Semmer AE, Lee MS, Harrison AR, Olsen TW. Hydroxychloroquine retinopathy screening. Br J Ophthalmol. 2008;92(12):1653-1655.

19. Kellner U, Renner AB, Tillack H. Fundus autofluorescence and mfERG for early detection of retinal alterations in patients using chloroquine/hydroxychloroquine. Invest Ophthalmol Vis Sci. 2006;47(8): 3531-3538.

20. Anderson C, Pahk P, Blaha GR, et al. Preferential hyperacuity perimetry to detect hydroxychloroquine retinal toxicity. Retina. 2009;29(8): $1188-1192$.

21. Chang WH, Katz BJ, Warner JE, Vitale AT, Creel D, Digre KB. A novel method for screening the multifocal electroretonogram in patients using hydroxychloroquine. Retina. 2008;28(10):1478-1486.

22. Lai TY, Chan WM, Li H, Lai RY, Lam DS. Multifocal electroretinographic changes in patients receiving hydroxychloroquine therapy. Am J Ophthalmol. 2005;140(5):794-807.

23. Lyons JS, Severns ML. Detection of early hydroxychloroquine retinal toxicity enhanced by ring ratio analysis of multifocal electroretinography. Am J Ophthalmol. 2007;143(5):801-809.

24. Maturi RK, Yu M, Weleber RG. Multifocal electroretinographic evaluation of long-term hydroxychloroquine users. Arch Ophthalmol. 2004;122(7):973-981.

25. Stepien KE, Han DP, Schell J, Godara P, Rha J, Carroll J. Spectraldomain optical coherence tomography and adaptive optics may detect hydroxychloroquine retinal toxicity before symptomatic vision loss. Trans Am Ophthalmol Soc. 2009;107:28-33.

26. Kelmenson AT, Brar VS, Murthy RK, Chalam KV. Fundus autofluorescence and spectral domain optical coherence tomography in early detection of Plaquenil maculopathy. Eur J Ophthalmol. 2009;20(4):785-788.

27. Brinkley JR Jr, Dubois EL, Ryan SJ. Long-term course of chloroquine retinopathy after cessation of medication. Am J Ophthalmol. 1979; 88(1):1-11.

\section{Dovepress}

PubMed Central and CAS, and is the official journal of The Society of Clinical Ophthalmology (SCO). The manuscript management system is completely online and includes a very quick and fair peer-review system, which is all easy to use. Visit http://www.dovepress.com/ testimonials.php to read real quotes from published authors. 